\title{
Association of cytomegalovirus diseases with newly developed myocardial infarction and congestive heart failure: data from a national population-based cohort
}

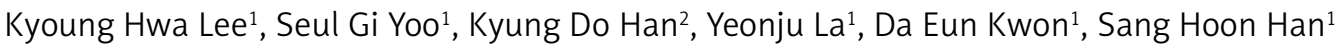

\author{
${ }^{1}$ Divison of Infectious Disease, Department of Internal Medicine, Yonsei University \\ College of Medicine, Seoul, Republic of Korea \\ 2Department of Biostatistics, Catholic University College of Medicine, Seoul, Republic \\ of Korea
}

Submitted: 5 November 2018; Accepted: 2 March 2019

Online publication: 19 March 2021

Arch Med Sci 2022; 18 (5): 1188-1198

DOI: https//doi.org/10.5114/aoms/105157

Copyright (๐) 2022 Termedia \& Banach

\section{Abstract}

Introduction: Anti-cytomegalovirus (CMV) IgG seropositive and/or titer are associated with a higher risk of cardiovascular diseases (CVD). However, it is not clear whether CMV end-organ disease may have a relation with development of CVD or chronic heart diseases.

Material and methods: In matched cohort study, the National Health Insurance Database covering 50 million people was used to identify 667 patients with CMV diseases and aged $\geq 20$ years between 2010 and 2014. 6,670 control subjects without CMV diseases were matched by age, sex, type 2 diabetes mellitus (DM), hypertension, dyslipidemia, and cohort entry year. Data on CMV disease and heart disease events of myocardial infarction (MI), congestive heart failure (CHF), and atrial fibrillation (AF) were retrieved. Previous events before CMV disease or cohort entry were excluded until January 2006. Subjects were followed until December 2015 in subjects without events and until date of events in subjects with events.

Results: The multivariate regression model adjusted by age, sex, low-income status, type 2 DM, hypertension, dyslipidemia, solid organ transplantation, and hematopoietic stem cell transplantation showed a significantly higher incidence rate of $\mathrm{MI}$ (odds ratio $(\mathrm{OR})=2.1,95 \%$ confidence intervals $(\mathrm{Cl}$ ): 1.0-4.5) and CHF (OR = 3.8, 95\% Cl: 2.1-6.8) but not $A F(O R=1.9,95 \% \mathrm{Cl}$ : 0.9-4.0) in patients with CMV disease. The age group of 40-64 years with CMV disease had the highest risk for new-onset CHF in this regression model $(\mathrm{OR}=9.4,95 \% \mathrm{Cl}: 4.12-21.44, p=0.029)$.

Conclusions: Symptomatic CMV tissue-invasive diseases were associated with a higher risk of new-onset $\mathrm{MI}$ and CHF.

Key words: cytomegalovirus, end-organ disease, myocardial infarction, heart failure, atrial fibrillation.

\section{Introduction}

In most cases, cytomegalovirus (CMV) infection is asymptomatically acquired in infancy and early childhood via breast milk or saliva and subsequently maintained in a latent status throughout the lifespan [1-3]. However, lytic replication through reactivation of latent CMV can result in diverse symptomatic tissue-invasive diseases with end-organ involvement, particularly fatal pneumonia or retinitis in severe immunocom-

\author{
Corresponding author: \\ Sang Hoon Han MD, PhD \\ Department of \\ Internal Medicine \\ Yonsei University \\ College of Medicine \\ 211 Eonju-ro \\ Gangnam-gu, Seoul \\ 06273, Republic of Korea \\ Phone: +82-2-2019-3319 \\ Fax: +82-2-3463-3882 \\ E-mail:shhan74@yuhs.ac
}


promised patients $[1,4]$. In addition, CMV-seropositive individuals without CMV diseases can have chronically significant CMV-specific CD8+ T-cell-mediated immune responses (CMV-CMI), indicating intermittent asymptomatic CMV reactivation and immune boosting [5-7]. Therefore, persistent inflation of CMV-CMI due to latent CMV infection can promote various chronic inflammatory diseases such as atherosclerosis, autoimmune disorders, and inflammatory bowel diseases [8-12]. The overt inflammatory necrotic CMV tissue-invasive diseases could lead to these chronic diseases through noteworthy intensification of the CMVCMI $[1,3,8]$.

Despite concerted efforts to control traditionally accepted modifiable risk factors including hypertension, type 2 non-insulin dependent diabetes mellitus (NIDDM), dyslipidemia, obesity, and smoking, the incidences of cardiovascular diseases (CVD), such as coronary heart disease (CHD), myocardial infarction (MI), and ischemic cerebral infarction, have been consistently increasing [13, 14]. Immune-mediated vascular endothelial damage in relation to currently unmodifiable contributors such as CMV or other unknown factors could be a possible cause of this epidemiologic phenomenon. Several studies suggest that the risk and mortality of heart diseases may increase with CMV infection due to chronic inflammation accompanied by T-cell associated immune senescence, endothelial cell injury, altered lipid profile, and vascular smooth muscle cell proliferation $[6,8$, 9, 15-23]. Conversely, some studies have reported that CMV infection was irrelevant to atherosclerosis and/or CVD [24-27]. Recent meta-analyses have demonstrated an association between CMV and heart diseases [28]. However, these results originated from seropositive status and/or titers in anti-CMV IgG tests, indicating previous CMV acquisition and humoral immunity [28]. It was not found that the tissue-invasive end-organ disease by active lytic replication of CMV may be related to higher incidence of CVD $[9,28]$. Thus, it would be worthwhile to determine whether symptomatic CMV tissue-invasive end-organ diseases, which are more relevant to overt inflammatory boosting, rather than silent CMV latent status by anti-CMV IgG serology, may increase the risk of heart diseases [29, 30].

In this study, we analyzed nationwide data pertaining to the entire population with CMV invasive diseases during a five-year period. We sought to determine whether cytopathic CMV replication that causes overt symptomatic organ disease is associated with the development of heart disease. Our results may constitute powerful evidence for supporting a relationship between CMV and heart diseases.

\section{Material and methods}

\section{The origin of data}

The South Korean National Health Insurance Service (KNHIS) is a mandatory state-operated system that was instituted to ensure universal healthcare. The KNHIS covers the entire South Korean population and provides health coverage by employment status. The low income group which accounts for about $2.8 \%$ of the total population is the beneficiary of the Medical Aid program, and the remaining population is divided into insurance for industrial workers and self-employed individuals [31]. Healthcare providers are required to submit all information pertaining to medical practice to the Health Insurance Review and Assessment (HIRA) to claim reimbursement of costs. The HIRA has systematically founded and managed a comprehensive data warehouse of beneficiaries, which is referred to as the National Health Insurance Database (NHID), for regularizing the National Health Insurance (NHIS) claims process. The NHID includes big data encompassing 1.3 trillion records with information regarding the results of medical diagnosis or treatment, long-term care insurance for the elderly, registration information on cancer or rare incurable diseases (RID), and status of medical institutions [32-34].

From 2005 onward, the KNHIS has implemented a policy to extend healthcare service to people experiencing economic difficulties due to excessive medical expenses related to RID and to help lower out-of-pocket expenses [32]. All CMV end-organ diseases are strictly registered as RID with specific codes and information sheets using predefined diagnostic criteria by the HIRA. We accessed the NHID for data from 2010 to 2014 as submitted to the National Health Insurance Sharing Service (NHISS) to analyze medical information pertaining to the entire Korean population [33-35]. This study was approved by the Institutional Review Board of the Gangnam Severance Hospital, Yonsei University College of Medicine, and relevant permission forms have been obtained from the NHISS.

\section{Definitions}

The diagnosis of CMV tissue-invasive end-organ diseases is based on several findings either in combination or separately as follows: 1) histopathologic features upon immunohistochemical staining for CMV, 2) measurement of pp65 antigen and/or detection of CMV in sterile body fluid or tissue using antigenemia test, culture, or nucleic acid amplification test [3, 4]. The unique V104 code for CMV diseases used in the enrollment of RID and submission to the HIRA completely corresponds with the B25 code within the International 
Statistical Classification of Diseases and Related Health Problems 10th Revision (ICD-10) codes, as presented in the 2016 online version by the World Health Organization (WHO) [36]. The B25 code includes all types of CMV end-organ diseases except for congenital CMV infection (P35.1) and cytomegaloviral mononucleosis (B27.1) [36].

The $\mathrm{CHA}_{2} \mathrm{DS}_{2}$-VASc score for risk stratification in atrial fibrillation (AF) was calculated using congestive heart failure, hypertension, age, DM, vascular disease, sex, and stroke/transient ischemic attack/thromboembolic events according to the American Heart Association guidelines [37]. We defined low-income status as the lower $25 \%$ percentile according to the annual household income based on results of the 2010 South Korea Population and Housing Census [35].

In this cohort study, events were defined as $\mathrm{MI}$ and/or congestive heart failure (CHF) and/or AF. The diseases were identified by ICD-10 diagnosis codes: acute or subsequent MI $(121,122), \mathrm{CHF}$ (150), AF (148.0, 148.1, 148.2, 148.9), hypertension (I10-I13, I15), type 2 NIDDM (E11), and dyslipidemia (E78 including E78.0-E78.9). The solid organ transplant (SOT) or hematopoietic stem cell transplant (HSCT) recipients of the high-risk group for CMV disease could have various underlying conditions attributing MI or CHF or AF. Therefore, we identified the SOT and HSCT recipients, who had received transplantation within 3 years from the index date, using unique codes for RID such as CMV disease, to adjust the confounding effects of CMV disease on development of heart diseases. We applied the RID codes of V084-V088 for kidney, liver, pancreas, heart, and lung transplantation, respectively. The V081-V083 codes were used for umbilical cord, autologous, and allogeneic HSCT, respectively. The point of cohort entry was assigned to the V104 code submission date for patients with CMV disease and to the matched date for individuals without CMV disease. The incidence rate (IR) per 1,000 patients was obtained by dividing the number of events with the total follow-up duration.

\section{Study population and design}

We conducted a case-controlled matched cohort study using the whole-population database. Using the V104 code, we identified 1,557 patients from the NHID who were diagnosed with CMV invasive disease between January 1, 2010 (cohort start date) and December 31, 2014, and followed them up until December 31, 2015 (cohort end date). We excluded 591 patients who were aged $<20$ years and 299 patients with a history of previous heart diseases such as $\mathrm{MI}$ and/or CHF and/or AF during the wash-out period (between January 2006 and the point of cohort entry). This exclusion criterion was used since heart diseases prior to CMV disease may affect the correlation between CMV and heart diseases. Finally, 667 patients with CMV invasive diseases were included in the case group, and the occurrence of newly developed heart disease was analyzed. The control group comprising 6,670 patients without CMV diseases (during the entire follow-up period and including any previous events during the washout period) was randomly selected at a $1: 10$ ratio matched by age, sex, type 2 NIDDM, hypertension, dyslipidemia and year of cohort entry (Figure 1 A). Therefore, our matched cohort enabled reliable identification of new-onset MI, CHF, and AF events. The follow-up was continued until occurrence of newly developed events in patients with events or until the cohort end date in patients without events in both groups. If the patients died after the occurrence of events, they were included in the event analyses and censored at date of death (Figure $1 \mathrm{~B}$ ).

\section{Statistical analysis}

Categorical data were expressed as numbers (percent), and continuous data were presented as means \pm standard deviation. Comparison between the matched groups was performed using the McNemar test and the paired $t$-test. Kaplan-Meier curves were constructed to analyze the incidence and probability of heart diseases according to the presence of CMV diseases. Multivariate logistic regression analyses using model 1 ( $M 1$, non-adjusted), model 2 ( $M 2$, adjusted by age and sex), model 3 (M3, adjusted by age, sex, low-income status, NIDDM, hypertension, and dyslipidemia), and model 4 (M4, adjusted by age, sex, low-income status, NIDDM, hypertension, dyslipidemia, SOT, and HSCT) were performed to evaluate the impact of CMV diseases on new-onset heart diseases. Statistical analyses were performed using the SAS program (version 9.2; SAS Institute, Cary, NC) and GraphPad Prism V6 (GraphPad Software, La Jolla, CA). A two-tailed $p$-value $<0.05$ was considered statistically significant.

\section{Results}

\section{Clinical characteristics of patients} in the matched cohort

The control group had significantly higher percentages of $\mathrm{MI}$ (control group vs. case group: $1.5 \%$ vs. $0.7 \%, p=0.035)$ and $\mathrm{CHF}(2.6 \%$ vs. $0.8 \%$, $p<0.001)$. The frequencies of SOT $(20.4 \%$ vs. $0.04 \%, p<0.001)$ or HSCT $(2.6 \%$ vs. $0 \%$, $p<0.001)$ recipients in the case group were significantly higher compared to the control group. The $\mathrm{CHA}_{2} \mathrm{DS}_{2}$-VASc score was also significantly higher in the case group than it was in the control group (case group vs. control group: $1.8 \pm 1.5$ vs. $1.5 \pm 1.4, p<0.001$ ). However, the frequency 
A

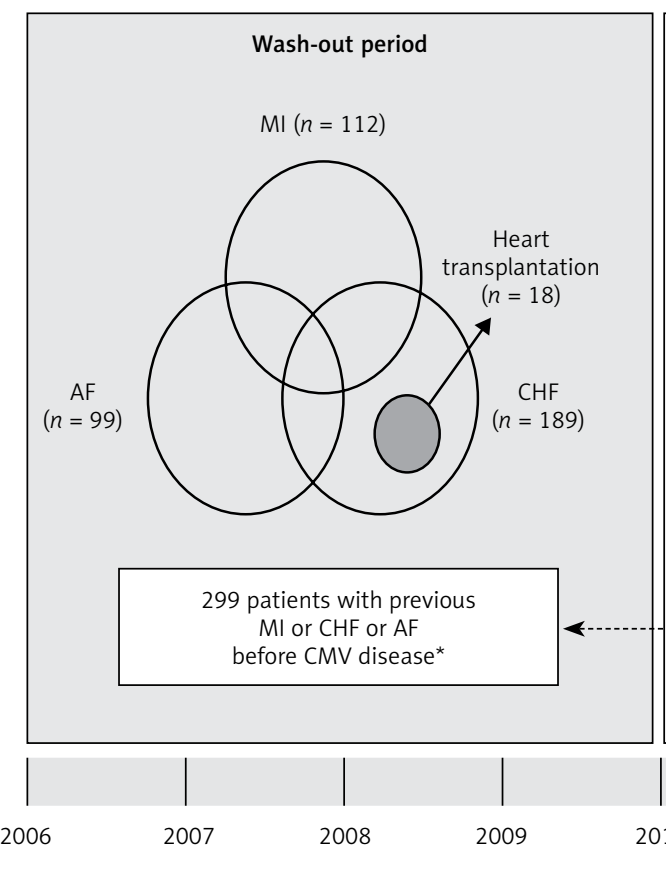

Start-date of cohort

Follow-up

End-date of cohort

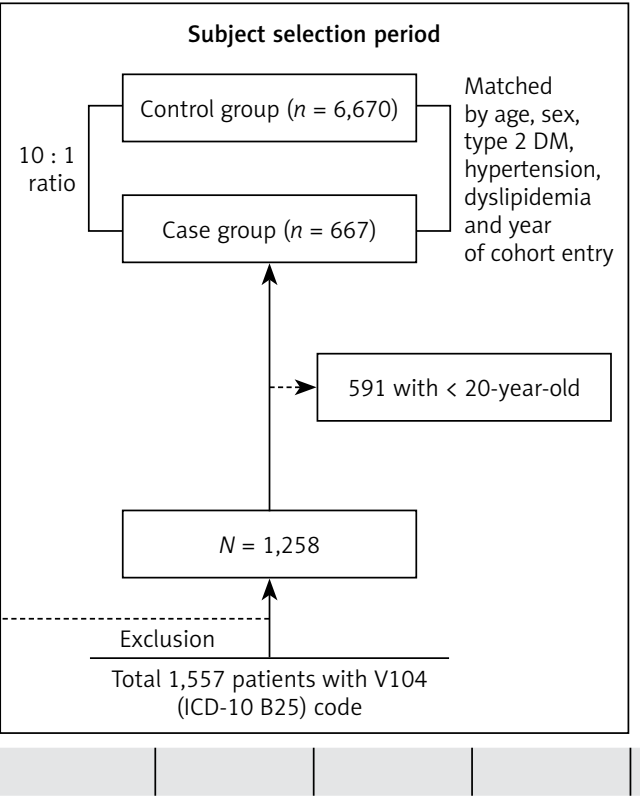

2011

2012

2013

2014

2015

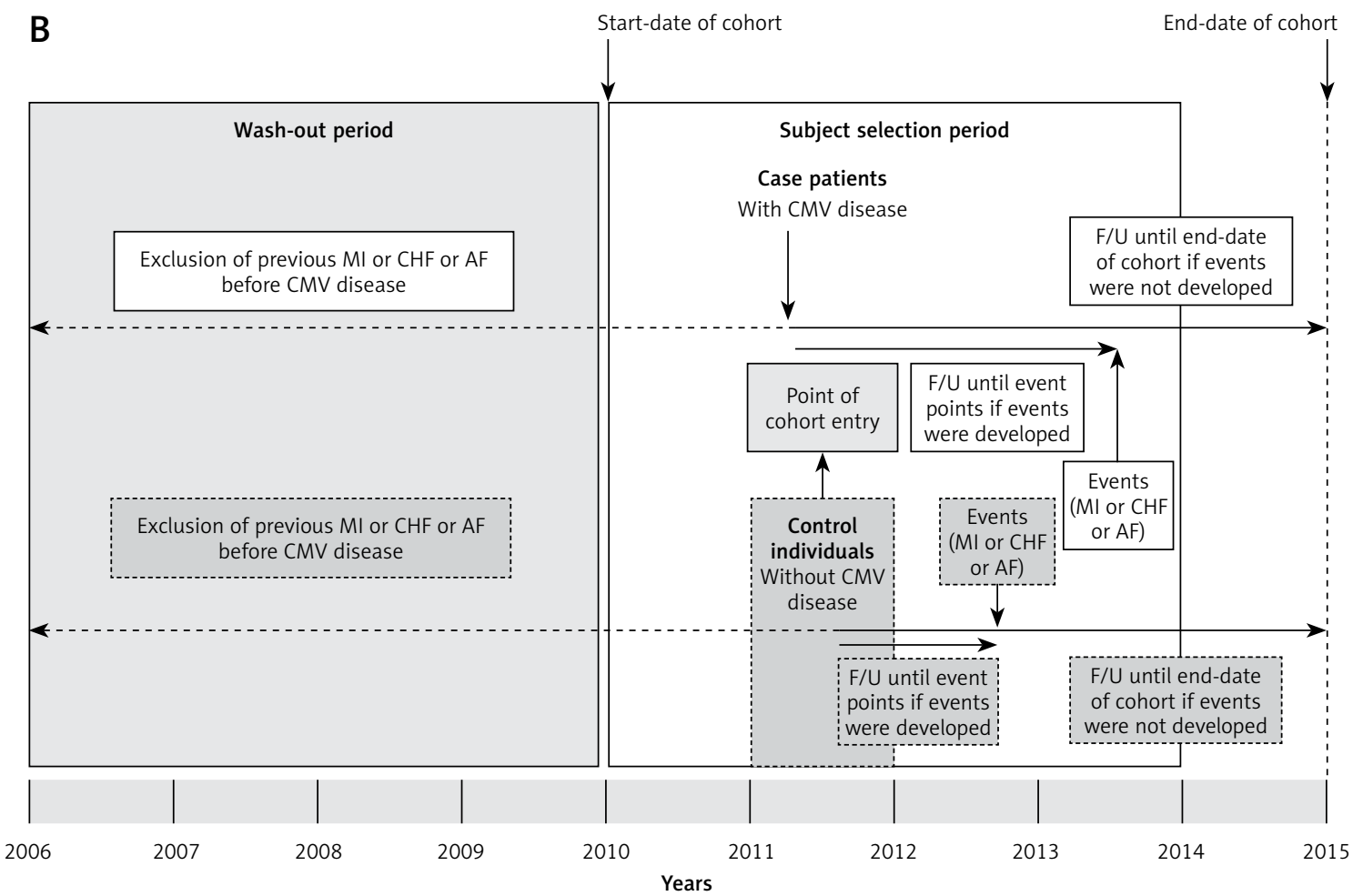

Figure 1. Schematic flow charts of study design and selection or follow-up of patients in case and control groups. A - Study design and patient selection. B - Schematic diagram of follow-up and exclusion of previous events in both groups

AF - atrial fibrillation, CHF - congestive heart failure, CMV - cytomegalovirus, DM - diabetes mellitus, F/U - follow-up, ICD-10 - the International Statistical Classification of Diseases and Related Health Problems $10^{\text {th }}$ Revision, MI - myocardial infarction, NHID - the National Health Insurance Database. 
of AF was not significantly different between the two groups (case group vs. control group: $1.4 \%$ vs. $0.9 \%, p=0.214$ ). The overall mean follow-up duration for each event was approximately 3 years in both groups (Table I).

\section{CMV disease and risk of newly developed $\mathrm{MI}, \mathrm{CHF}$, and $\mathrm{AF}$}

The IR/1,000 patients of $\mathrm{MI}$ in the case group was approximately two-fold higher than that in the control group as per M3 with an odds ratio (OR) of 2.2 (95\% confidence interval $(\mathrm{Cl})$ : 1.1-4.2) and as per M4 with an OR of $2.1(95 \% \mathrm{Cl}$ : 1.0-4.5). The IR/1,000 patients of CHF in the case group was also up to three-fold higher than that in the control group (M3, 3.7 (2.1-6.3) and M4, 3.8 (2.1-6.8)) (Table II). During the maximal 6-year follow-up period, the incidence probabilities of $\mathrm{MI}$ $(p=0.023)$ and CHF $(p<0.001)$ were significantly higher in the case group than those in the control group (Figures $2 \mathrm{~A}, \mathrm{~B}$ ).

In contrast, the IR of AF showed a lower OR (1.6-1.9) than that of $\mathrm{MI}$ or CHF; the IR of AF was not significantly different between the case and the control groups (95\% Cl: $\mathrm{M} 3,0.8-3.3$ and M4, 0.9-4.0) (Table II). The incidence probability of newly developed AF was similar between the two groups as per Kaplan-Meier curve analysis $(p=0.169)$ (Figure 2 C).

\section{The risk difference of newly developed heart diseases in age and sex subgroup analysis}

The ORs for MI were higher in men than in women (M4, 2.3 vs. 1.7), and the OR was also higher in the 40-64 years age group compared to that in the $\geq 65$ years age group ( $M 4,2.6$ vs. 2.0). In the case of CHF, the ORs were higher in women than in men (M4, 4.2 vs. 3.4), and higher in the 40-64 years age group than in the $\geq 65$ years age group (M4, 9.4 vs. 1.7). The middle-aged (40-64-yearold) patients in the case group had the higher ORs and statistically significant $95 \% \mathrm{Cls}$ for CHF ( $p$-values for subgroup interaction $=0.029$ ). However, there were no statistically significant differences in the IRs of MI, CHF, and AF associated with CMV disease in the overall sex and age subgroup analysis except CHF in age group (Table III).

Table I. Comparison of clinical characteristics between adult individuals with and without CMV disease in a matched cohort

\begin{tabular}{|c|c|c|c|}
\hline \multirow[t]{2}{*}{ Characteristics } & \multicolumn{2}{|c|}{ CMV disease } & \multirow[t]{2}{*}{$P$-value } \\
\hline & Yes $(n=667)$ & No $(n=6,670)$ & \\
\hline Sex, male & $368(55.17)$ & $3,680(55.17)$ & $>0.999^{*}$ \\
\hline Age [years]: & $50.58 \pm 15.34$ & $50.58 \pm 15.33$ & $>0.999^{\dagger}$ \\
\hline $20-39$ & $165(24.74)$ & $1,650(24.74)$ & \\
\hline $40-64$ & $381(57.12)$ & $3,810(57.12)$ & \\
\hline$\geq 65$ & $121(18.14)$ & $1,210(18.14)$ & \\
\hline Low income status & $179(26.84)$ & $1,553(23.28)$ & $0.039^{*}$ \\
\hline Type 2 NIDDM & $146(21.89)$ & $1,460(21.89)$ & $>0.999^{*}$ \\
\hline Hypertension & $260(38.98)$ & $2,600(38.98)$ & $>0.999^{*}$ \\
\hline Dyslipidemia & $164(24.59)$ & $1,640(24.59)$ & $>0.999^{*}$ \\
\hline SOT & $136(20.39)$ & $3(0.04)$ & $<0.001^{*}$ \\
\hline $\mathrm{HSCT}$ & $17(2.55)$ & $0(0.00)$ & $<0.001^{*}$ \\
\hline $\mathrm{CHA}_{2} \mathrm{DS}_{2}$-VASc Score & $1.80 \pm 1.46$ & $1.51 \pm 1.39$ & $<0.001^{\dagger}$ \\
\hline Acute or subsequent MI & $10(1.50)$ & $49(0.73)$ & $0.035^{*}$ \\
\hline Congestive heart failure & $17(2.55)$ & $52(0.78)$ & $<0.001^{*}$ \\
\hline Atrial fibrillation & $9(1.35)$ & $58(0.87)$ & $0.214^{*}$ \\
\hline Death & $66(9.9)$ & $165(2.47)$ & $<0.001^{*}$ \\
\hline \multicolumn{4}{|l|}{ Follow-up duration ${ }^{\ddagger}$ [years]: } \\
\hline Myocardial infarction & $3.07 \pm 1.60$ & $3.25 \pm 1.52$ & $0.005^{\dagger}$ \\
\hline Congestive heart failure & $3.06 \pm 1.62$ & $3.25 \pm 1.52$ & $0.002^{\dagger}$ \\
\hline Atrial fibrillation & $3.07 \pm 1.61$ & $3.24 \pm 1.52$ & $0.007^{\dagger}$ \\
\hline Death & $3.09 \pm 1.60$ & $3.26 \pm 1.52$ & $0.005^{\dagger}$ \\
\hline
\end{tabular}

Data are expressed as number (percent) or mean $\pm S D .{ }^{*}$ McNemar test; ${ }^{\dagger}$ Paired $t$-test; ${ }^{\ddagger}$ Between cohort entry point and development of each event in patients with new-onset MI, CHF, AF, or death, or between cohort entry point and cohort end date in patients without development of each event. CMV - cytomegalovirus, HSCT - hematopoietic stem cell transplantation, NIDDM - non-insulin dependent diabetes mellitus, $\mathrm{MI}$ - myocardial infarction, SOT - solid organ transplantation. 
Table II. Multivariate logistic regression models to examine the effect of cytomegalovirus (CMV) disease in adults

\begin{tabular}{|c|c|c|c|c|c|c|c|c|c|}
\hline \multirow[t]{2}{*}{ Outcomes } & \multirow{2}{*}{$\begin{array}{c}\text { CMV } \\
\text { disease }\end{array}$} & \multirow[t]{2}{*}{ No. } & \multirow[t]{2}{*}{ Events } & \multirow{2}{*}{$\begin{array}{l}\text { Total follow-up } \\
\text { duration [years] }\end{array}$} & \multirow[t]{2}{*}{$I R^{*}$} & \multicolumn{4}{|c|}{ Odds ratio $(95 \% \mathrm{Cl})$} \\
\hline & & & & & & Model 1 & Model 2 & Model 3 & Model 4 \\
\hline \multirow[t]{2}{*}{ MI } & No & 6,670 & 49 & $21,644.42$ & 2.264 & 1 (Ref.) & 1 (Ref.) & 1 (Ref.) & 1 (Ref.) \\
\hline & Yes & 667 & 10 & $2,048.02$ & 4.883 & $\begin{array}{c}2.16 \\
(1.03-4.08)\end{array}$ & $\begin{array}{c}2.21 \\
(1.05-4.17)\end{array}$ & $\begin{array}{c}2.23 \\
(1.07-4.22)\end{array}$ & $\begin{array}{c}2.14 \\
(1.01-4.52)\end{array}$ \\
\hline \multirow[t]{2}{*}{$\mathrm{CHF}$} & No & 6,670 & 52 & $21,681.55$ & 2.400 & 1 (Ref.) & 1 (Ref.) & 1 (Ref.) & 1 (Ref.) \\
\hline & Yes & 667 & 17 & $2,040.14$ & 8.333 & $\begin{array}{c}3.47 \\
(1.95-5.87)\end{array}$ & $\begin{array}{c}3.62 \\
(2.03-6.12)\end{array}$ & $\begin{array}{c}3.73 \\
(2.09-6.31)\end{array}$ & $\begin{array}{c}3.79 \\
(2.10-6.84)\end{array}$ \\
\hline \multirow[t]{2}{*}{$A F$} & No & 6,670 & 58 & $21,605.37$ & 2.685 & 1 (Ref.) & 1 (Ref.) & 1 (Ref.) & 1 (Ref.) \\
\hline & Yes & 667 & 9 & $2,048.60$ & 4.393 & $\begin{array}{c}1.63 \\
(0.75-3.12)\end{array}$ & $\begin{array}{c}1.69 \\
(0.78-3.24)\end{array}$ & $\begin{array}{c}1.70 \\
(0.79-3.26)\end{array}$ & $\begin{array}{c}1.89 \\
(0.90-3.96)\end{array}$ \\
\hline
\end{tabular}

Model 1 - non-adjusted, Model 2 - age-and sex-adjusted, Model 3 -age-, sex-, low income status-, type 2 NIDDM-, hypertensionand dyslipidemia-adjusted, Model 4 -age-, sex-, low income status-, type 2 NIDDM-, hypertension-, dyslipidemia-, SOT- and HSCTadjusted. "per 1,000 patients. AF - atrial fibrillation, CHF - congestive heart failure, Cl - confidence interval, CMV - cytomegalovirus, HSCT - hematopoietic stem cell transplantation, IR - incidence rate, $M I$ - myocardial infarction, NIDDM - non-insulin dependent diabetes mellitus, No. - number, Ref. - reference, SOT - solid organ transplantation.

A

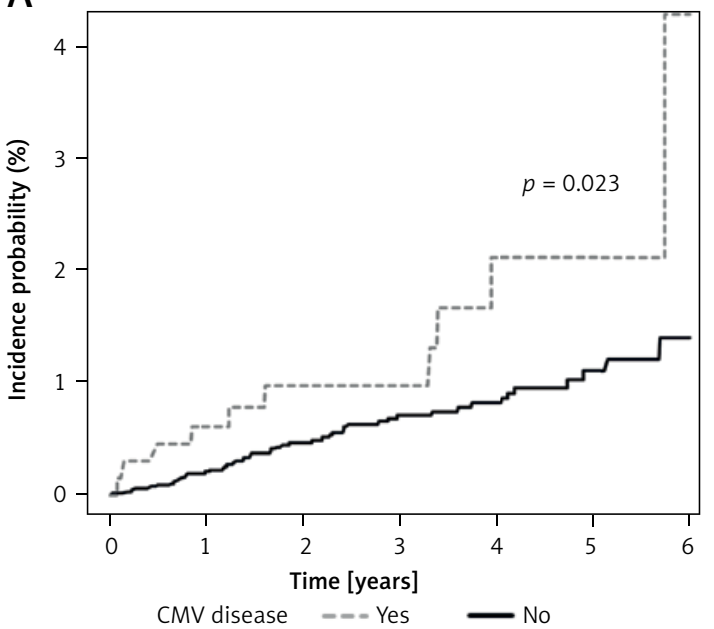

C

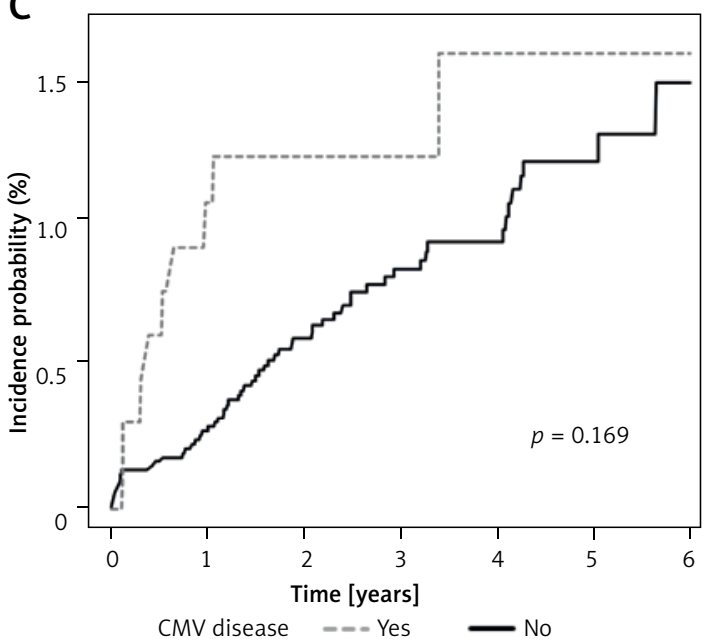

\section{Discussion}

This nationwide study aimed to clarify the relationship between CMV tissue-invasive end-organ

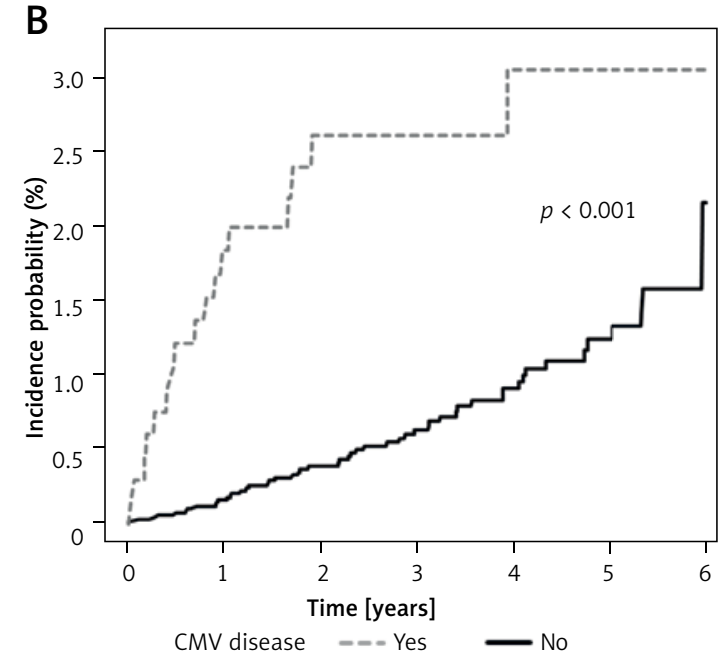

Figure 2. Kaplan-Meier curves for incidence probability of heart diseases according to tissue-invasive end-organ disease caused by cytomegalovirus. A - Myocardial infarction. B - Congestive heart failure. $\mathbf{C}$ - Atrial fibrillation

CMV - cytomegalovirus.

diseases and new-onset heart diseases during a 6-year follow-up period. Previous studies with various study designs and participant characteristics have evaluated different aspects of asso- 


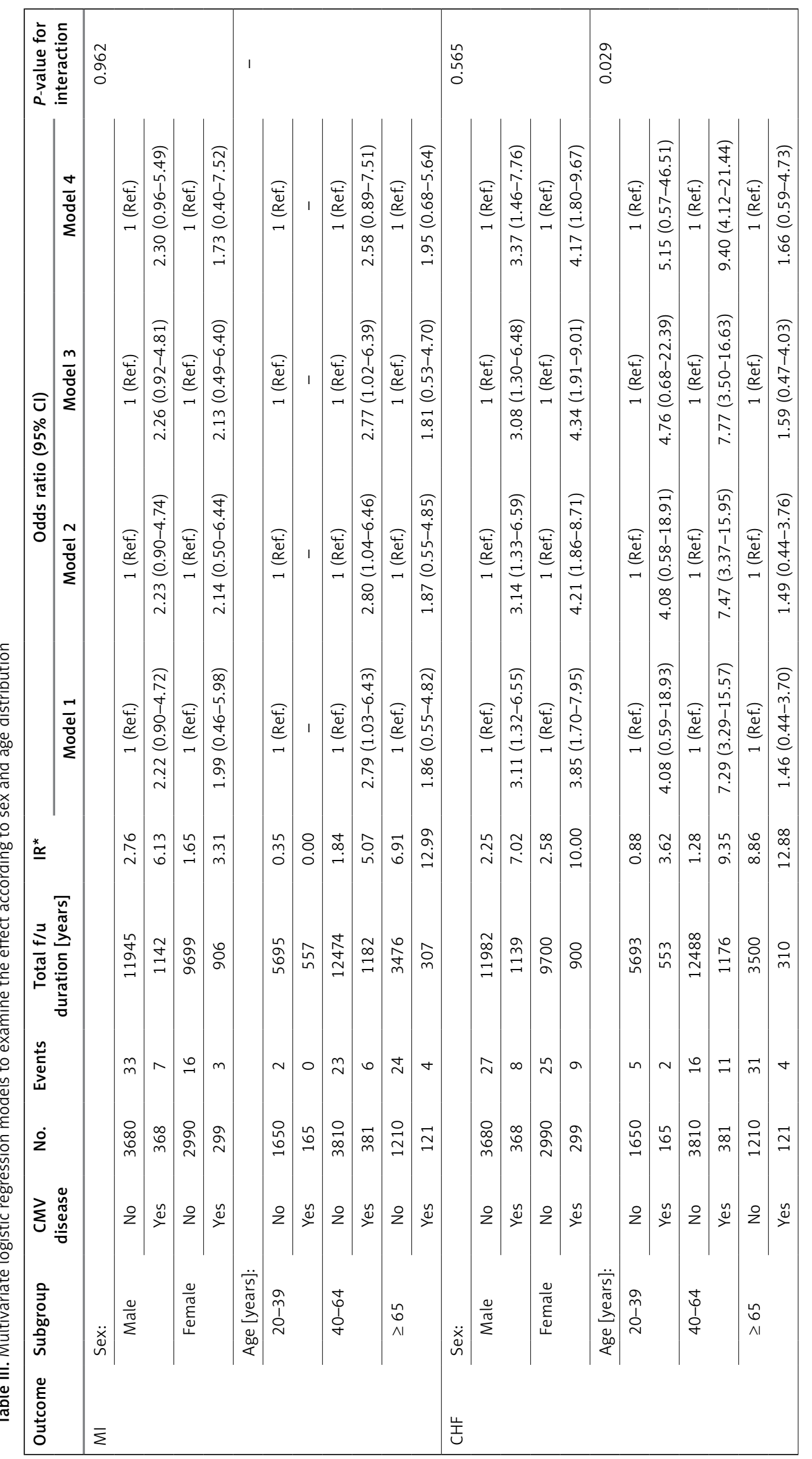




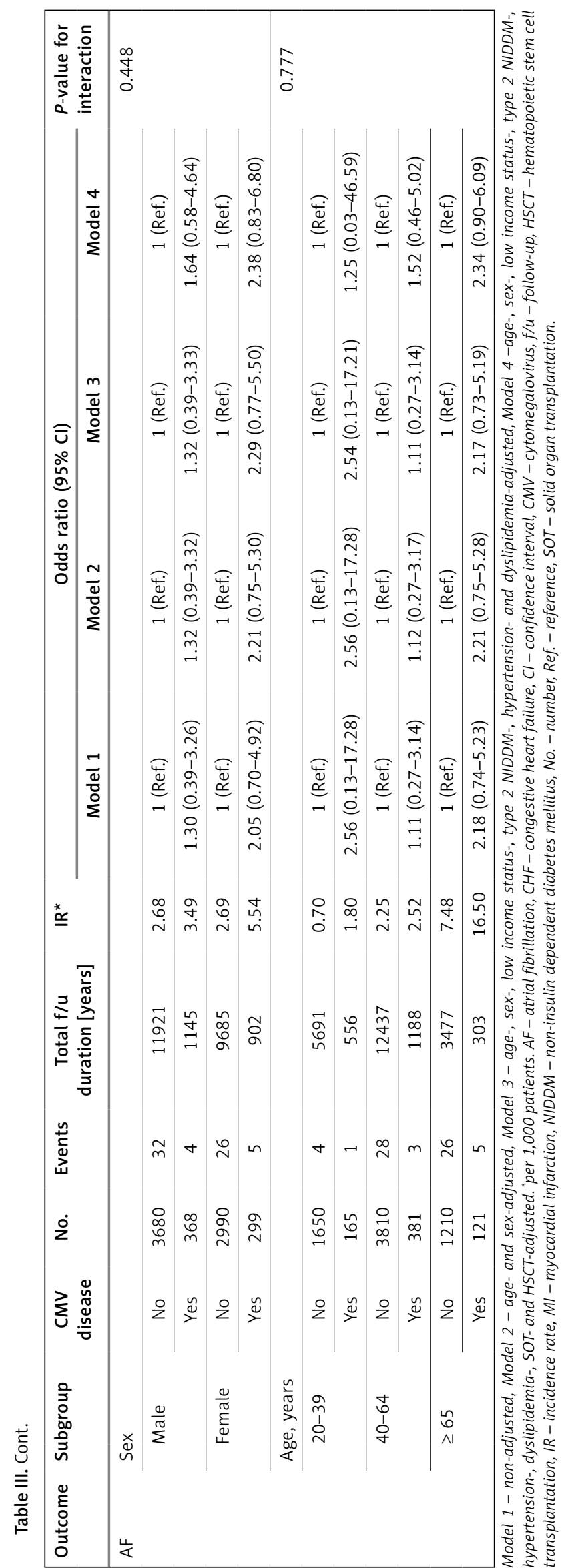

ciations between CMV and CVD [18, 22, 24-27], and a recent meta-analysis performed with 10 prospective studies showed that CMV infection significantly increased the risk of CVD with low relative risk (RR) of 1.22 [28]. However, by confining the analysis to three studies of ischemic heart disease (IHD), including coronary artery disease, $\mathrm{CHD}$, and $\mathrm{MI}$, the overall RR of CMV infection was not statistically significant $(p=0.145)$ [28]. Despite the large number of participants and CVD events, the most important drawback of studies including this meta-analysis was the simplification of CMV infection as just a positive result or a high titer in an anti-CMV IgG serology test [28]. In our first study, we defined CMV status as tissue-invasive organ disease resulting in strong immunologic effects; the results of that study revealed that the development of CMV diseases was associated with a $200-400 \%$ higher risk of new-onset MI or CHF.

In addition, using a definite cytopathic inflammatory manifestation to define symptomatic CMV disease, this study has several unique strengths, as follows: (1) a long wash-out period to exclude previous heart diseases in groups with and without CMV diseases, which ensured the exploration of a relationship between CMV disease and $\mathrm{MI}$ or CHF; (2) precise ICD-10 code-based detection of $\mathrm{Ml}$ or CHF events, in contrast to CHD or coronary artery disease diagnoses; this allowed for effective evaluation of the clinical impact of active CMV replication on CVD; (3) the retrieval of data on subjects and events from a database covering the entire national population of 50 million; this ensured greater reliability of data and statistics; (4) the relatively small number of events from this well-stratified matched cohort retrieved from a large database; this may have reduced the proportion of misdiagnoses of CMV and heart diseases.

Interestingly, the analysis of our cohort which excluded heart transplantation recipients showed that CMV disease increased the risk of $\mathrm{CHF}$ but did not significantly affect AF. CMV replication in heart transplantation recipients is one of the major risk factors of graft dysfunction and CHF $[4,17]$. However, the epidemiologic association between $\mathrm{CMV}$ and $\mathrm{CHF}$ or $\mathrm{AF}$ in the general population has not been evaluated. IHD is the most important and common cause of and predisposing factor for CHF [38]. Therefore, the chronic or strong inflammatory processes induced by CMV invasive disease may promote CHF caused by IHD-associated systolic dysfunction. In contrast, it is unclear whether any association exists between AF and CD8 ${ }^{+}$T-cell-mediated CMI [39]. This new finding, in contrast to the association between CMV and AF, would support our hypothesis about the relationship between CMV-inducted chronic inflammation and inflammatory heart diseases such as $\mathrm{MI}$ and $\mathrm{CHF}$. 
Table IV. Distribution for incidence of cytomegalovirus tissue-invasive end-organ disease according to ICD-10 codes in the entire-population database

\begin{tabular}{|lccccc|}
\hline ICD-10 codes & \multicolumn{5}{c|}{ Year } \\
\cline { 2 - 6 } & 2010 & 2011 & 2012 & 2013 & 2014 \\
\hline B25 (Cytomegalovirus disease) & 2 & 0 & 1 & 0 & 0 \\
\hline B25.0 (Cytomegaloviral pneumonitis) & 14 & 14 & 14 & 16 & 17 \\
\hline B25.1 (Cytomegaloviral hepatitis) & 20 & 10 & 10 & 19 & 26 \\
\hline B25.2 (Cytomegaloviral pancreatitis) & 0 & 0 & 0 & 0 & 0 \\
\hline B25.8 (Other cytomegaloviral diseases) & 56 & 77 & 93 & 87 & 123 \\
\hline B25.9 (Cytomegaloviral disease, unspecified) & 67 & 105 & 87 & 137 & 193 \\
\hline Total (N = 1,188) & 159 & 206 & 205 & 259 & 359 \\
\hline
\end{tabular}

Data are represented as numbers, obtained from the Korean Health Insurance Review and Assessment Service claims data warehouse. ICD-10 - International Statistical Classification of Diseases and Related-Health Problems $10^{\text {th }}$ Revision.

Table V. Frequencies of solid organ transplantation recipients with cytomegalovirus disease or infection according to ICD-10 codes between 2010 and 2015 in the entire-population database

\begin{tabular}{|lcc|}
\hline & \multicolumn{2}{c|}{ Patients with CMV disease } \\
\hline ICD-10 codes & Total individuals & SOT recipients \\
\hline B25 (Cytomegalovirus disease) & 3 & $0(0.0)$ \\
\hline B25.0 (Cytomegaloviral pneumonitis) & 89 & $6(6.7)$ \\
\hline B25.1 (Cytomegaloviral hepatitis) & 101 & $4(4.0)$ \\
\hline B25.2 (Cytomegaloviral pancreatitis) & 4 & $0(0.0)$ \\
\hline B25.8 (Other cytomegaloviral diseases) & 485 & $80(16.5)$ \\
\hline B25.9 (Cytomegaloviral disease, unspecified) & 621 & $193(31.1)$ \\
\hline Total & 1,303 & $283(21.7)$ \\
\hline
\end{tabular}

Data are presented as number (percent). CMV - cytomegalovirus, ICD-10 - International Statistical Classification of Diseases and RelatedHealth Problems $10^{\text {th }}$ Revision, SOT - solid organ transplantation.

In addition to the immunologic mechanisms underlying the impact of CMV-inducted inflammation on atherosclerosis [9], subacute CMV infection inhibits $P 53$ gene product activity and promotes smooth muscle cell proliferation and restenosis in coronary angioplasty patients [40]. Viperin is an interferon-inducible antiviral protein derived from CMV, which promotes inflammatory responses of the host; the activity of this protein may explain the impact of CMV infection on $\mathrm{MI}$ and $\mathrm{CHF}[41,42]$. Viperin interacts with mitochondria to reduce cellular adenosine triphosphate production, inhibits mitochondrial apoptosis, and ultimately induces inflammatory processes [41]. Natural killer cells, nuclear factor $\kappa \mathrm{B}$, and pro-inflammatory cytokines, such as tumor necrosis factor- $\alpha$ and interleukin- 6 , also contribute to the development of atherosclerosis [9]. Further studies pertaining to the pathogenesis of atherosclerosis in active CMV replication conditions, including CMV DNAemia or tissue-invasive end-organ diseases, but excluding the non-replicative latent status of seropositivity, will be warranted to verify the following hypothesis: upregulation of inflammation (CMV-CMI) induced by the active production of CMV virions could potentiate the process of CVD development.
This study has some limitations that should be considered: (1) This is a retrospective observational study with a short mean follow-up time of 3 years. In our study, Kaplan-Meier curve analysis showed that the incidence of $\mathrm{MI}, \mathrm{CHF}$, and $\mathrm{AF}$ increased progressively throughout the follow-up period in the control group without CMV diseases. These findings adequately demonstrate that our cohort was effectively matched. However, the occurrence of CHF increased rapidly within 1 year after the development of CMV diseases. The incidence of new-onset MI varied significantly 3 years after the development of CMV diseases. (2) The data were based on NHID, and the presence of diseases was designated using diagnostic codes. The NHID in South Korea is under surveillance by the HIRA and has a high level of accuracy, but wrong or missed diagnoses cannot be ruled out due to the large size of the database. Also, the diagnosis of CMV diseases based on the V104 code may have included symptomatic CMV invasive disease cases with unclear histopathologic findings. However, the detailed data for each ICD-10 code of CMV end-organ diseases in our entire cohort showed low incidences of CMV pneumonitis, hepatitis, and pancreatitis (Table IV). These three end-organ diseases are relatively rare 
in real clinical practice and confirmatory pathologic-based diagnosis of these conditions is difficult since it is not easy to obtain tissue samples. The majority of CMV diseases ( $\geq 85 \%$ ) involved codes B25.8 or B25.9, indicating other organs including the gastrointestinal tract. (3) We analyzed the association of three representative heart diseases - MI, CHF, and AF - with CMV diseases. A future prospective study of the relationship of non-cardiogenic vascular diseases such as peripheral vascular diseases with symptomatic CMV diseases is warranted. Due to relatively high inaccuracy of diagnostic codes, we were unable to perform this type of study. (4) Our entire cohort included approximately $20 \%$ of severely immunocompromised solid organ transplantation (SOT) recipients (Table V). The effect of active CMV replication on the outcome of diseases is considered highly significant in SOT [4]. In addition, we could not acquire detailed information about the immunocompromised status or diseases directly associated with CMV end-organ diseases for all the participants. Therefore, it was not possible to adjust for all of the known contributable confounding factors for MI or CHF. (5) There were relatively few events of $\mathrm{MI}, \mathrm{CHF}$, and $\mathrm{AF}$ in both groups. However, given the low IR of CMV end-organ diseases, it may be difficult to conduct a well-designed prospective study to gather enough CMV and heart disease events. (6) The cohort was constituted almost entirely of participants of South Korean origin, because the data were derived from the Korean medical insurance system. Therefore, ethnic differences could not be analyzed. (7) Our observational cohort study may potentially have informational bias arising from the difference of follow-up loss between two groups.

Despite these limitations, our results are consistent with a recent meta-analysis of 10 prospective studies, and our hypothesis is supported by results from several in-vitro studies. Thus, our study confirms that CMV, regardless of latent non-replicative, active lytic (tissue-invasive end-organ disease), asymptomatic, or symptomatic status, is a definite risk factor of IHD and atherosclerosis [9, $10,22,23,28,40,42]$. To additionally reduce the burden of ischemic vascular diseases, tailored preventive or therapeutic strategies with vaccines or anti-CMV drugs in particularly high risk patients should be explored in the near future. Immune monitoring by QuantiFERON-CMV, intracellular cytokine staining or other similar methods would be helpful to evaluate and stratify patients based on risk of CMV-associated CVD [43].

In conclusion, this whole-population-based matched cohort study suggests that symptomatic CMV invasive organ disease is a contributing factor for new-onset MI or CHF with a high incidence.

\section{Conflict of interest}

The authors declare no conflict of interest.

\section{References}

1. Gandhi MK, Khanna R. Human cytomegalovirus: clinical aspects, immune regulation, and emerging treatments. Lancet Infect Dis 2004; 4: 725-38.

2. Manicklal S, Emery VC, Lazzarotto T, Boppana SB, Gupta RK. The "silent" global burden of congenital cytomegalovirus. Clin Microbiol Rev 2013; 26: 86-102.

3. Griffiths P, Baraniak I, Reeves M. The pathogenesis of human cytomegalovirus. J Pathol 2015; 235: 288-97.

4. Razonable RR, Humar A. Cytomegalovirus in solid organ transplantation. Am J Transplant 2013; 13 Suppl 4: 93-106.

5. Poole E, Sinclair J. Sleepless latency of human cytomegalovirus. Med Microbiol Immunol 2015; 204: 421-9.

6. Elwenspoek MMC, Sias K, Hengesch X, et al. T cell immunosenescence after early life adversity: association with cytomegalovirus infection. Front Immunol 2017; 8: 1263 .

7. Weltevrede M, Eilers R, de Melker HE, van Baarle D. Cytomegalovirus persistence and T-cell immunosenescence in people aged fifty and older: a systematic review. Exp Gerontol 2016; 77: 87-95.

8. Broadley I, Pera A, Morrow G, Davies KA, Kern F. Expansions of cytotoxic CD4(+)CD28(-) T cells drive excess cardiovascular mortality in rheumatoid arthritis and other chronic inflammatory conditions and are triggered by CMV infection. Front Immunol 2017; 8: 195.

9. Du Y, Zhang G, Liu Z. Human cytomegalovirus infection and coronary heart disease: a systematic review. Virol J 2018; 15: 31.

10. Lee YL, Liu CE, Cho WL, et al. Presence of cytomegalovirus DNA in leucocytes is associated with increased oxidative stress and subclinical atherosclerosis in healthy adults. Biomarkers 2014; 19: 109-13.

11. Halenius A, Hengel $H$. Human cytomegalovirus and autoimmune disease. Biomed Res Int 2014; 2014: 472978.

12. Siegmund B. Cytomegalovirus infection associated with inflammatory bowel disease. Lancet Gastroenterol Hepatol 2017; 2: 369-76.

13. Benjamin EJ, Blaha MJ, Chiuve SE, et al. Heart disease and stroke statistics-2017 update: a report from the American Heart Association. Circulation 2017; 135: e146-603.

14. Yatsuya H, Li Y, Hilawe EH, et al. Global trend in overweight and obesity and its association with cardiovascular disease incidence. Circ J 2014; 78: 2807-18.

15. Kaptoge S, Di Angelantonio E, Lowe G, et al. C-reactive protein concentration and risk of coronary heart disease, stroke, and mortality: an individual participant meta-analysis. Lancet 2010; 375: 132-40.

16. Corrado E, Novo S. Role of inflammation and infection in vascular disease. Acta Chir Belg 2005; 105: 567-79.

17. Grattan MT, Moreno-Cabral CE, Starnes VA, Oyer PE, Stinson EB, Shumway NE. Cytomegalovirus infection is associated with cardiac allograft rejection and atherosclerosis. JAMA 1989; 261: 3561-6.

18. Nikitskaya E, Lebedeva A, Ivanova O, et al. Cytomegalovirus-productive infection is associated with acute coronary syndrome. J Am Heart Assoc 2016; 5: e00375.

19. Pawelec G, Derhovanessian E. Role of CMV in immune senescence. Virus Res 2011; 157: 175-9. 
20. Bauer ME, Fuente Mde L. The role of oxidative and inflammatory stress and persistent viral infections in immunosenescence. Mech Ageing Dev 2016; 158: 27-37.

21. Redeker A, Remmerswaal EBM, van der Gracht ETI, et al. The contribution of cytomegalovirus infection to immune senescence is set by the infectious dose. Front Immunol 2017; 8: 1953.

22. Spyridopoulos I, Martin-Ruiz C, Hilkens C, et al. CMV seropositivity and T-cell senescence predict increased cardiovascular mortality in octogenarians: results from the Newcastle 85+ study. Aging Cell 2016; 15: 389-92.

23. Yu HT, Park S, Shin EC, Lee WW. T cell senescence and cardiovascular diseases. Clin Exp Med 2016; 16: 257-63.

24. Adler SP, Hur JK, Wang JB, Vetrovec GW. Prior infection with cytomegalovirus is not a major risk factor for angiographically demonstrated coronary artery atherosclerosis. J Infect Dis 1998; 177: 209-12.

25. Haider AW, Wilson PW, Larson MG, et al. The association of seropositivity to Helicobacter pylori, Chlamydia pneumoniae, and cytomegalovirus with risk of cardiovascular disease: a prospective study. J Am Coll Cardiol 2002; 40: 1408-13.

26. Fagerberg B, Gnarpe J, Gnarpe H, Agewall S, Wikstrand J. Chlamydia pneumoniae but not cytomegalovirus antibodies are associated with future risk of stroke and cardiovascular disease: a prospective study in middle-aged to elderly men with treated hypertension. Stroke 1999; 30: 299-305.

27. Ridker PM, Hennekens CH, Stampfer MJ, Wang F. Prospective study of herpes simplex virus, cytomegalovirus, and the risk of future myocardial infarction and stroke. Circulation 1998; 98: 2796-9.

28. Wang H, Peng G, Bai J, et al. Cytomegalovirus infection and relative risk of cardiovascular disease (ischemic heart disease, stroke, and cardiovascular death): a metaanalysis of prospective studies up to 2016. J Am Heart Assoc 2017; 6: e005025.

29. Clement M, Humphreys IR. Cytokine-mediated induction and regulation of tissue damage during cytomegalovirus infection. Front Immunol 2019; 10: 78.

30. Sissons JG, Wills MR. How understanding immunology contributes to managing CMV disease in immunosuppressed patients: now and in future. Med Microbiol Immunol 2015; 204: 307-16.

31. Kwon S. Thirty years of national health insurance in South Korea: lessons for achieving universal health care coverage. Health Policy Plan 2009; 24: 63-71.

32. Kim S, Kwon S. Impact of the policy of expanding benefit coverage for cancer patients on catastrophic health expenditure across different income groups in South Korea. Soc Sci Med 2015; 138: 241-7.

33. Kim JA, Yoon S, Kim LY, Kim DS. Towards actualizing the value potential of Korea Health Insurance Review and Assessment (HIRA) data as a resource for health research: strengths, limitations, applications, and strategies for optimal use of HIRA data. J Korean Med Sci 2017; 32: 718-28

34. Lee J, Lee JS, Park SH, Shin SA, Kim K. Cohort profile: the National Health Insurance Service-National Sample Cohort (NHIS-NSC), South Korea. Int J Epidemiol 2017; 46: e15.

35. National Health Insurance Service. National Health Insurance \& Long-Term Care Insurance System in Republic of Korea [Internet]. The National Health Insurance Service Republic of Korea,; 2018. [cited. Available from: https://www.nhis.or.kr/static/html/wbd/g/a/wbdga0704.html.
36. World Health Organization. International Statistical Classification of Diseases and Related Health Problems 10th Revision (ICD-10) [Internet]. World Health Organization,; 2016. [cited. Available from: http://apps.who. int/classifications/icd10/browse/2016/en.

37. January CT, Wann LS, Alpert JS, et al. 2014 AHA/ACC/HRS guideline for the management of patients with atrial fibrillation: executive summary: a report of the American College of Cardiology/American Heart Association Task Force on practice guidelines and the Heart Rhythm Society. Circulation 2014; 130: 2071-104

38. Yancy CW, Jessup M, Bozkurt B, et al. 2013 ACCF/AHA guideline for the management of heart failure: a report of the American College of Cardiology Foundation/ American Heart Association Task Force on practice guidelines. Circulation 2013; 128: e240-327.

39. Hu YF, Chen YJ, Lin YJ, Chen SA. Inflammation and the pathogenesis of atrial fibrillation. Nat Rev Cardiol 2015; 12: 230-43.

40. Speir E, Modali R, Huang ES, et al. Potential role of human cytomegalovirus and p53 interaction in coronary restenosis. Science 1994; 265: 391-4.

41. Seo JY, Yaneva R, Hinson ER, Cresswell P. Human cytomegalovirus directly induces the antiviral protein viperin to enhance infectivity. Science 2011; 332: 1093-7.

42. Olofsson PS, Jatta K, Wagsater D, et al. The antiviral cytomegalovirus inducible gene 5/viperin is expressed in atherosclerosis and regulated by proinflammatory agents. Arterioscler Thromb Vasc Biol 2005; 25: e113-6.

43. Han SH. Immunological prediction of cytomegalovirus (CMV) replication risk in solid organ transplantation recipients: approaches for regulating the targeted antiCMV prevention strategies. Infect Chemother 2017; 49: 161-75. 